\title{
Cardiac catheterization and percutaneous intervention procedures on extracorporeal membrane oxygenation support
}

\author{
Cesar Y. Guerrero-Miranda ${ }^{1,2,3}$, Shelley A. Hall ${ }^{1,2,3}$ \\ ${ }^{1}$ Annette C. and Harold C. Simmons Transplant Institute, Baylor Scott \& White Research Institute, Dallas, TX, USA; ${ }^{2}$ Center for Advanced Heart \\ and Lung Disease, Baylor University Medical Center, Dallas, TX, USA; ${ }^{3}$ Department of Internal Medicine, Texas A \& M University Health Science \\ Center, Dallas, TX, USA \\ Correspondence to: Cesar Y. Guerrero-Miranda, MD. Center for Advanced Heart and Lung Disease, Baylor University Medical Center, 3410 Worth \\ Street, Suite 250, Dallas, TX 75246, USA. Email: Cesar.GuerreroMiranda@BSWHealth.org.
}

\begin{abstract}
Extracorporeal membrane oxygenation (ECMO) is used to support critically ill patients when conventional therapies have failed. ECMO has been available for four decades and has gained use as a rescue therapy in severe refractory hypoxic disorders and in patients with refractory cardiogenic shock (RCS). Over recent years, several percutaneous cardiac interventions and implant devices have been developed that are now used frequently in conjunction with ECMO in order to maintain organ perfusion. Here, we review the literature on VA-ECMO cannulation location, the use of VA-ECMO in interventions (e.g., coronary interventions and structural heart interventions) and percutaneous cardiac device implantation in VA-ECMO recipients with RCS.
\end{abstract}

Keywords: Extracorporeal membrane oxygenation (ECMO); cardiogenic shock; heart failure (HF); cardiac catheterization; percutaneous devices

Submitted Jun 30, 2018. Accepted for publication Nov 21, 2018.

doi: $10.21037 /$ acs.2018.11.08

View this article at: http://dx.doi.org/10.21037/acs.2018.11.08

\section{Introduction}

Extracorporeal membrane oxygenation (ECMO), also known as extracorporeal life support (ECLS), is used to support critically ill patients when conventional therapies have failed. ECMO can be used as a venovenous ( $\mathrm{VV}$ ECMO) circuit for artificial pulmonary bypass or as a venoarterial (VA-ECMO) circuit for systemic circulation restoration. The ECMO system consists of bypass cannulae, an extracorporeal centrifugal pump (e.g., TandemHeart, Centrimag, Rotaflow or Biomedicus pump), a heat exchanger and a hollow-fiber microporous membrane oxygenator (1). ECMO has been available for four decades and was initially used primarily in neonates and in cases of respiratory distress (2). It has gained use as a rescue therapy in severe refractory hypoxic disorders and in patients with refractory cardiogenic shock (RCS). According to the Extracorporeal Life Support Organization (ELSO) International Registry, 78,397 children and adults were supported with ECMO between 1989 and 2016, of whom $70 \%$ were successfully weaned off ECMO and 58\% survived hospital discharge. Over recent years, several percutaneous cardiac interventions and implant devices have been developed that are now used frequently in conjunction with ECMO in order to maintain organ perfusion. Here, we review the literature on VA-ECMO cannulation location, the use of VA-ECMO in interventions (e.g., coronary interventions and structural heart interventions), including diagnosis and indication for use of ECMO support, and percutaneous cardiac device implantation in VA-ECMO recipients with RCS.

\section{VA-ECMO cannulation location}

There are two principal types of VA-ECMO cannulation: central and peripheral. Central cannulation is mostly used in post-cardiotomy shock and often utilizes the existing cannulation from the cardiopulmonary bypass (CPB). A single cannula, located in the right atrium (RA) or bicaval cannulation through the RA and then joined to create a single drainage channel, is used to extract venous 
blood and send it to the pump (3). Direct central, short and large-bore venous cannulas allow a more effective cardiac decompression compared to peripheral venous cannulation (4). Oxygenated blood is returned to the body via arterial cannulation, usually inserted in the ascending aorta, with less concern for retrograde flow or upper body hypoxia compared with peripheral VAECMO (4). Other arterial cannulation alternatives include the innominate or right subclavian or axillary arteries (5). Cannulas can be tunneled to allow the chest to be closed. A major disadvantage is the requirement to re-open the chest for decannulation, exposing the patient to potential complications like bleeding, thrombosis or infections.

Peripheral VA-ECMO may be placed at the bedside without fluoroscopy guidance. Venous cannulation uses either an infrahepatic inferior vena cava cannula or a large 21-25 French multistage cannula inserted in the femoral vein with its tip in the RA. Oxygenated blood is returned via the arterial cannula inserted in the femoral artery, typically a short 17-21 French, with the tip ending in the iliac artery. The main advantage of peripheral ECMO is the easier cannulation or decannulation process. Potential disadvantages include upper body hypoxia, aortic root thrombosis, increase LV afterload and limb ischemia (4). Cheng et al. published a meta-analysis of 1,866 patients' post-ECMO after cardiogenic shock and reported incidences of $16.9 \%$ for lower extremity ischemia, $10.3 \%$ for compartment syndrome and $4.7 \%$ for amputation (6). To prevent these complications, the insertion of a distally directed 6-10 French catheter for limb perfusion is recommended. This approach has been found to be associated with a decrease in the rate of limb ischemia.

\section{Use of VA-ECMO in RCS}

RCS, the most severe expression of heart failure (HF), is preceded by myocardial contractile dysfunction, which leads to inadequate tissue perfusion and, in turn, can result in multi-organ failure. Acute myocardial infarction (MI) and subsequent ischemic heart disease are the cause of approximately $80 \%$ of the cases of RCS (7). The 2013 American Heart Association (AHA)/American College of Cardiology (ACC) Guidelines for the Management of STElevation Myocardial Infarction (STEMI) assigns a level $\mathrm{IIb} / \mathrm{c}$ indication for left ventricular assist devices (LVADs) in RCS (8), including both TandemHeart and VA-ECMO.

Over the past decade, the use of temporary mechanical circulatory support (TMCS) has increased rapidly in the treatment of patients with RCS and is associated with reduced hospital costs and, in some cases, reduced inhospital mortality (9). TMCS devices intend to restore systemic perfusion and prevent further end-organ damage until the insult that resulted in cardiogenic shock is addressed (10). In cases of RCS with biventricular failure and reduced systemic oxygenation, VA-ECMO can provide full cardiopulmonary support and is thus used as a rescue TMCS. VA-ECMO systems can generate flow of more than $4.5 \mathrm{~L} / \mathrm{min}$. Their use is recommended up to 7 days and requires systemic anticoagulation.

A report from the ELSO registry showed that RCS was the most common diagnosis associated with VAECMO and that $42 \%$ of these patients survived to hospital discharge (11). Guenther et al. observed a 30-day survival rate of $49 \%$ in patients with RCS. The 5 -year actuarial survival was $65.2 \% \pm 7.9 \%$ in a multi-institutional experience (12). Those with post-cardiotomy RCS did considerably worse, with an overall survival rate to hospital discharge of $30.8 \%$ (13). Although a meta-analysis on the use of ECMO for various etiologies of cardiogenic shock has not been performed, it appears that the best survival rate of VA-ECMO use in cardiogenic shock is found in adults with acute fulminant myocarditis (up to $83 \%$ successful ECMO weaning and survival to hospital discharge) (14). In patients with RCS and peripheral VA-ECMO, it is important to allow the heart to remain ejecting in order to maintain coronary flow and cerebral circulation and to avoid left heart overdistention, thrombus formation and potential systemic embolization. Left ventricular (LV) contractility can be achieved by using a low-dose inotrope, by decreasing VA-ECMO flow, or by reducing systemic vascular resistance with vasodilators. The contractility can easily be monitored using arterial line pulsatility. As the venous blood is drained to the ECMO pump, most of the pulmonary circulation is bypassed, except for blood returned to the $\mathrm{LV}$ from the sinus venosus, bronchial and Thebesian circulation. Thus, VA-ECMO can provide $80 \%$ of the cardiac circulation. Usually, VA-ECMO flow is maintained at $\geq 2 \mathrm{~L} / \mathrm{min} / \mathrm{m}^{2}$. An adequate systemic blood flow and oxygen supply ensure that hematocrit remains above $33 \%$, arterial partial oxygen pressure $\left(\mathrm{PaO}_{2}\right)>100 \mathrm{mmHg}$ and mean arterial pressure $\geq 65 \mathrm{mmHg}$ (1). Clinically, a normal capillary refill time, normalization of arterial blood $\mathrm{pH}$, reduction of lactates and increased urine output are signs of improved systemic perfusion (1).

The hemodynamic condition of the $\mathrm{LV}$ of patients in cardiogenic shock, either due to acute decompensated 
HF or to large acute MI, are characterized by elevated $\mathrm{LV}$ end-diastolic volume and $\mathrm{LV}$ end-diastolic pressure, markedly reduced LV stroke volume and LV stroke work, but with increased myocardial oxygen demand and potentially impaired coronary blood flow due to elevated wall stress and hypotension. Bavaria et al demonstrated in healthy sheep hearts that VA-ECMO reduces myocardial oxygen consumption and LV wall stress. However, in post ischemic, dilated hearts, LV wall stress increases and it is proportional to the VA-ECMO flow, mostly due to an increase in afterload (15). Increased left atrial pressure, even more relevant if associated with severe mitral regurgitation, results in pulmonary congestion, pulmonary edema and in extreme cases, pulmonary hemorrhage, which can lead to irreversible pulmonary failure. Boulate $e t$ al. observed early acute lung injury (ALI) in $27 \%$ of patients who underwent transition from VA-ECMO to long-term mechanical circulatory support (MCS) with mortality up to $87 \%$ while on long-term MCS support (16).

In comparison with the Impella (Abiomed Inc., Danvers, MA, USA) and Tandem-Heart (TandemLife, Pittsburgh, PA, USA) percutaneous MCS, VA-ECMO without $L V$ venting increases $L V$ systolic and diastolic pressures while reducing $\mathrm{LV}$ stroke volume, with a net increase in arterial elastance (17). Different $L V$ venting approaches may be considered: percutaneous [such as intra-aortic balloon pumps (IABP), Impella or TandemHeart device or atrial septostomy], surgical (transapical LV cannulation or direct LA cannulation) or non-invasive (by allowing the $\mathrm{LV}$ contractility and aortic valve opening with inotrope or reducing VA-ECMO flow) (18). The use of percutaneous cardiac devices, like Impella or Tandem-Heart as a strategy for $L V$ venting has the advantage that these devices help unload the LV, decrease filling pressures and improve coronary perfusion (17). In a recent retrospective analysis, the use of EC-VAD strategy (i.e., a combination of VA-ECMO and a percutaneous microaxial LVAD) was associated with a significant decrease in pulmonary artery pressures and improved mixed venous saturation value $\left(\mathrm{SvO}_{2}\right)$ and $\mathrm{PaO}_{2} / \mathrm{FiO}_{2}$ ratio, markers for ALI 24 hours post-implantation, compared with VA-ECMO alone (19). Takeda et al. published their experience with 25 patients who underwent a novel minimally invasive external VAD with a magnetically levitated centrifugal pump via apicoaxillary cannulation. For 17 (68\%) of these patients, a VA-ECMO circuit was added by femoral vein cannulation and an oxygenator. There was a $68 \%$ survival to the next destination (recovery, durable MCS or heart transplantation) (20).

\section{VA-ECMO as a rescue intervention in acute MI or high risk percutaneous coronary artery intervention}

Standardized criteria to define what constitutes a "high risk population" for percutaneous coronary intervention (PCI) do not exist. The decision is often based on a combination of clinical, anatomic and hemodynamic criteria. Clinical criteria may include cardiogenic shock occurring within 24 hours or at the start of PCI, left ventricular ejection fraction (LVEF) $\leq 30 \%$, Killip class II-IV on presentation, PCI within 24 hours after cardiac arrest, STEMI or acute coronary syndrome (ACS) complicated with hemodynamic instability or ventricular arrhythmias. Anatomic criteria may include unprotected left main disease or left main equivalent, planned PCI in substrate of severe multivessel coronary artery disease (CAD) or PCI of a vessel or graft that supply a large territory. Hemodynamic criteria include evidence of cardiogenic shock (sustained systolic blood pressure (SBP) $<90 \mathrm{mmHg}$ or required use of vasopressors, cardiac index $<2.2 \mathrm{~L} / \mathrm{min} / \mathrm{m}^{2}$, with evidence of end-organ hypoperfusion and pulmonary capillary wedge pressure $>15 \mathrm{mmHg})(7,21)$.

The 2011 ACCF/AHA/SCAI Guidelines for PCI recommend elective insertion of percutaneous TMCS devices for hemodynamic support as adjunct to high risk PCI (class IIb, level of evidence C). The percutaneous devices that historically were implanted by the interventional cardiologist in the cardiac catheterization lab, such as the IABP, Impella or Tandem-Heart, offer only partial hemodynamic support. VA-ECMO has the advantages of providing full hemodynamic support ( $>4.5 \mathrm{~L} / \mathrm{min}$ flow), independent of any unstable heart rhythm, reducing carbon dioxide and adding oxygen to venous blood before return to the arterial circulation, bypassing pulmonary circulation with potential use for several days (1). A limiting factor for its use as rescue therapy is the implantation complexity, requiring technical skills with a multidisciplinary approach usually including a cardiologist, cardiothoracic surgeon, anesthesiologist, cardiac catheterization personnel and a perfusionist. Therefore, VA-ECMO is not frequently used in the cardiac cath lab. Contraindications to ECMO include age $>75$ years, life expectancy $<1$ year, significant aortic regurgitation, severe peripheral vascular disease, contraindications to systemic anticoagulation and neurological injury. Sheu et al. demonstrated that early 
VA-ECMO can significantly reduce 30-day mortality and prolong in-hospital survival in patients with STEMI complicated with profound cardiogenic shock (22).

\section{VA-ECMO and elective coronary artery bypass grafting (CABG)}

Cardiohelp ECLS (MAQUET, Cardiopulmonary AG, Germany) is the world's smallest portable heart-lung support system. It is currently available mostly for ECMO patients who need transportation. It may also be used in clinical settings outside the operating room. Some centers have successfully used Cardiohelp in elective CABG procedures with excellent patient outcomes. Haneya et al. from the University Medical Center in Regensburg, Germany compared a series of 50 patients undergoing elective CABG surgery using conventional $\mathrm{CPB}$ versus 50 patients using the Cardiohelp ECLS. They found significant decreases in the intra-operative blood transfusion requirement, time on mechanical ventilation, post-operative inotropic support, length of stay in the ICU and postoperative levels of lactic acid and creatinine kinase with the Cardiohelp device compared to the conventional $\mathrm{CPB}$ circuit (23).

\section{VA-ECMO in transcatheter aortic valve implantation (TAVI)}

The emerging population of patients with compromised cardiac function undergoing PCI or valvular therapies, especially the adult population with inoperable aortic valvular disease, typically requires cardiac support during percutaneous aortic valve replacement or TAVI. VAECMO is mostly used as rescue intervention in emergency situations, or as a peri-operative prophylactic. However, the long-term use of VA-ECMO in this elderly and frail population undergoing TAVI is probably futile and inappropriate.

Seco et al. showed a series of 11 patients on VAECMO support ( $\mathrm{n}=8$ prophylactic, $\mathrm{n}=3$ rescue) among a cohort of 100 patients who underwent TAVI between 2009 and 2013 at Royal Prince Alfred Hospital in Sydney, Australia. Compared with non-VA-ECMO, VA-ECMOsupported TAVI patients had a significantly higher mean EuroSCORE, but overall mortality was not significantly different ( $9 \%$ vs. $2 \% ; \mathrm{P}>0.05$ ) and post procedure outcomes were similar (24).

Another center (University of Regensburg, Germany) reported the use of VA-ECMO as rescue intervention for TAVI complications in 8 of 131 cases (including ventricular perforation, cardiogenic shock and ventricular tachycardia). VA-ECMO was then used prophylactically in nine patients who were deemed very high risk. The median EuroSCORE in this subgroup was considerably higher compared to the remaining TAVI cohort. Comparing prophylactic use to rescue VA-ECMO, procedural success and 30-day mortality were $100 \%$ vs. $44 \%(\mathrm{P}=0.03)$ and $0 \%$ vs. $44 \%(\mathrm{P}=0.02)$, respectively (25).

\section{Use of VA-ECMO in post-infarct ventricular septal defect (PI-VSD)}

PI-VSD is a rare but life-threatening complication after MI (26). More prevalent in infarcts due to complete occlusion of the left anterior descending coronary artery, in females and older patients, PI-VSD's prevalence has reduced from $1-2 \%$ to $0.2 \%$ in the post-thrombolysis and PCI area; nonetheless, with an elevated morbidity and mortality (worse in those cases treated medically only). Despite successful surgical or percutaneous repair, 30-day mortality is approaching $94 \%$ in the current era. Timing for repair is also controversial, often being delayed due to concern of fragile and necrotic VSD tissue which would produce a poor success rate, so use of LVAD or ECMO are important to maintain hemodynamic support. The role of rescue VA-ECMO in either unrepaired PI-VSD patients with rapid deterioration and multi-organ failure, or post-cardiotomy repaired VSD patients with failure to remove from $\mathrm{CPB}$, are commonly used but published only as small series of cases with variable success rates (27). Femoral-femoral cannulation is mostly used in PI-VSD with cardiogenic shock and central cannulation is often approached in failed post-CBP patients. Various case reports have suggested that extracorporeal support may be an option to allow hemodynamic stability, thus allowing a delayed closure approach (28).

\section{Discussion}

The use of VA-ECMO as rescue or back-up cardiopulmonary support has opened a new horizon for high-risk PCI and structural heart procedures in patients complicated with cardiogenic shock and underlines complex multivessel disease or multiple other comorbidities, who were previously relegated to conservative medical management. For special populations, such as pediatric patients with 
myocardial disease (including myocarditis, HF or congenital cardiomyopathies), the options for circulatory support are very limited. The most commonly used approach in children is VA-ECMO. Despite of the lack of prospective randomized controlled trials in this area, VA-ECMO is a reasonable MCS alternative for hemodynamic support during high-risk cardiac procedures. The limitations of the data reviewed here include the small size of many studies and their retrospective nature, making comparisons among these percutaneous MCS technologies difficult.

\section{Conclusions}

Adequate recovery of a failing heart, either due to RCS, after MI or due to fatal arrhythmias, largely depends on the immediate short-term hemodynamic stabilization and completeness of coronary revascularization, if indicated. Extracorporeal support via VA-ECMO provides excellent rescue hemodynamic control and can be inserted peripherally without delay. Patients can be supported with ECMO for a few days or weeks and then can be disconnected when myocardial recovery is adequate or bridged to long-term VADs or transplantation. Additionally, optimal LV loading conditions in ECMO patients can be achieved with the addition of percutaneously delivered MCS devices. Similarly, VA-ECMO has been demonstrated to be useful in maintaining a stable condition for diagnostic and therapeutic procedures in high risk CAD or structural heart disease like aortic stenosis or ischemic ventricular septal defects. Despite this benefit, large studies are necessary to confirm the advantage of the addition of VAECMO in surgical coronary revascularization and repair of structural heart disease.

\section{Acknowledgements}

None.

\section{Footnote}

Conflicts of Interest: Dr. Hall is a consultant for Abiomed, Inc. The other author has no conflicts of interest to declare.

\section{References}

1. Sangalli F, Patroniti N, Pesenti A, et al. editors. ECMO-Extracorporeal Life Support in Adults. Milan: Springer, 2014.
2. Gregoric ID. Mechanical circulatory support in acute heart failure. Tex Heart Inst J 2012;39:854-5.

3. Pierce EC II. Extracorporeal circulation for open-heart surgery. Springfield, Ill: Charles C. Thomas, 1970.

4. Jayaraman AL, Cormican D, Shah P, et al. Cannulation strategies in adult veno-arterial and veno-venous extracorporeal membrane oxygenation: Techniques, limitations, and special considerations. Ann Card Anaesth 2017;20:S11-8.

5. Sarkar M, Prabhu V. Basics of cardiopulmonary bypass. Indian J Anaesth 2017;61:760-7.

6. Cheng R, Hachamovitch R, Kittleson M, et al. Complications of extracorporeal membrane oxygenation for treatment of cardiogenic shock and cardiac arrest: a meta-analysis of 1,866 adult patients. Ann Thorac Surg 2014;97:610-6.

7. Thiele H, Ohman EM, Desch S, et al. Management of cardiogenic shock. Eur Heart J 2015;36:1223-30.

8. O'Gara PT, Kushner FG, Ascheim DD, et al. 2013 ACCF/ AHA guideline for the management of ST-elevation myocardial infarction: a report of the American College of Cardiology Foundation/American Heart Association Task Force on Practice Guidelines. J Am Coll Cardiol 2013;61:e78-140.

9. Stretch R, Sauer CM, Yuh DD, et al. National trends in the utilization of short-term mechanical circulatory support: incidence, outcomes, and cost analysis. J Am Coll Cardiol 2014;64:1407-15.

10. Guerrero-Miranda CY, Hall SA. Dog Model Holds Promise for Early Mechanical Unloading in Patients With Acute Myocardial Infarction. Circ Heart Fail 2018;11:e004972.

11. Thiagarajan RR, Barbaro RP, Rycus PT, et al. Extracorporeal Life Support Organization Registry International Report 2016. ASAIO J 2017;63:60-7.

12. Lorusso R, Centofanti P, Gelsomino S, et al. Venoarterial Extracorporeal Membrane Oxygenation for Acute Fulminant Myocarditis in Adult Patients: A 5-Year Multi-Institutional Experience. Ann Thorac Surg 2016;101:919-26.

13. Khorsandi M, Dougherty S, Bouamra O, et al. Extracorporeal membrane oxygenation for refractory cardiogenic shock after adult cardiac surgery: a systematic review and meta-analysis. J Cardiothorac Surg 2017;12:55.

14. Kawahito K, Murata S, Yasu T, et al. Usefulness of extracorporeal membrane oxygenation for treatment of fulminant myocarditis and circulatory collapse. Am J Cardiol 1998;82:910-1. 
15. Bavaria JE, Ratcliffe MB, Gupta KB, et al. Changes in left ventricular systolic wall stress during biventricular circulatory assistance. Ann Thorac Surg 1988;45:526-32.

16. Boulate D, Luyt CE, Pozzi M, et al. Acute lung injury after mechanical circulatory support implantation in patients on extracorporeal life support: an unrecognized problem. Eur J Cardiothorac Surg 2013;44:544-9.

17. Briceno N, Kapur NK, Perera D. Percutaneous mechanical circulatory support: current concepts and future directions. Heart 2016;102:1494-507.

18. Kapur NK, Esposito M. Hemodynamic support with percutaneous devices in patients with heart failure. Heart Fail Clin 2015;11:215-30.

19. Takeda K, Garan AR, Ando M, et al. Minimally invasive CentriMag ventricular assist device support integrated with extracorporeal membrane oxygenation in cardiogenic shock patients: a comparison with conventional CentriMag biventricular support configuration. Eur J Cardiothorac Surg 2017;52:1055-61.

20. Takeda K, Garan AR, Topkara VK, et al. Novel minimally invasive surgical approach using an external ventricular assist device and extracorporeal membrane oxygenation in refractory cardiogenic shock. Eur J Cardiothorac Surg 2017;51:591-6.

21. Myat A, Patel N, Tehrani S, et al. Percutaneous circulatory assist devices for high-risk coronary intervention. JACC Cardiovasc Interv 2015;8:229-44.

22. Sheu JJ, Tsai TH, Lee FY, et al. Early extracorporeal membrane oxygenator-assisted primary percutaneous coronary intervention improved 30-day clinical outcomes in patients with ST-segment elevation myocardial infarction complicated with profound cardiogenic shock. Crit Care Med 2010;38:1810-7.

23. Haneya A, Philipp A, Camboni D, et al. Successful coronary artery bypass grafting with the aid of a portable minimized extracorporeal life support system. ASAIO J 2012;58:337-42.

24. Seco M, Forrest P, Jackson SA, et al. Extracorporeal Membrane Oxygenation for Very High-risk Transcatheter Aortic Valve Implantation. Heart Lung Circ 2014;23:957-62.

25. Husser O, Holzamer A, Philipp A, et al. Emergency and prophylactic use of miniaturized veno-arterial extracorporeal membrane oxygenation in transcatheter aortic valve implantation. Catheter Cardiovasc Interv 2013;82:E542-51.

26. Crenshaw BS, Granger CB, Birnbaum Y, et al. Risk factors, angiographic patterns, and outcomes in patients with ventricular septal defect complicating acute myocardial infarction. GUSTO-I (Global Utilization of Streptokinase and TPA for Occluded Coronary Arteries) Trial Investigators. Circulation 2000;101:27-32.

27. McLaughlin A, McGiffin D, Winearls J, et al. VenoArterial ECMO in the Setting of Post-Infarct Ventricular Septal Defect: A Bridge to Surgical Repair. Heart Lung Circ 2016;25:1063-6.

28. Suder B, Janik L, Wasilewski G, et al. Post-myocardial infarction ventricular septal defect. Is it better to operate on a fresh infarction or to wait? A case study. Kardiochir Torakochirurgia Pol 2016;13:39-41.
Cite this article as: Guerrero-Miranda CY, Hall SA. Cardiac catheterization and percutaneous intervention procedures on extracorporeal membrane oxygenation support. Ann Cardiothorac Surg 2019;8(1):123-128. doi: 10.21037/ acs.2018.11.08 